\title{
On the augmented Biot-JKD equations with Pole-Residue representation of the dynamic tortuosity
}

\author{
Miao-Jung Yvonne $\mathrm{Ou}^{\dagger},^{1}$ Hugo J. Woerdeman 倜, \\ †Department of Mathematical Sciences, University of Delaware, Newark, DE 19716, USA, \\ mou@udel.edu \\ ${ }^{\ddagger}$ Department of Mathematics, Drexel University, Philadelphia, PA 19104, USA, \\ hugo@math.drexel.edu
}

Dedicated to our colleague and friend Joe Ball

\begin{abstract}
In this paper, we derive the augmented Biot-JKD equations, where the memory terms in the original Biot-JKD equations are dealt with by introducing auxiliary dependent variables. The evolution in time of these new variables are governed by ordinary differential equations whose coefficients can be rigorously computed from the JKD dynamic tortuosity function $T^{D}(\omega)$ by utilizing its Stieltjes function representation derived in [20], where an approach for computing the pole-residue representation of the JKD tortuosity is also proposed. The two numerical schemes presented in the current work for computing the poles and residues representation of $T^{D}(\omega)$ improve the previous scheme in the sense that they interpolate the function at infinite frequency and have much higher accuracy than the one proposed in [20].
\end{abstract}

\section{Introduction}

Poroelastic composites are two-phase composite materials consisted of elastic solid frames with fluidsaturated pore space. The study of poroelasticity plays an important role in biomechanics, seismology and geophysics due to the nature of objects of research in these fields, eg. fluid saturated rocks, sea ice and cancellous bone. It is of great interest for modeling wave propagation in these materials. When the wave length is much higher than the scale of the microstructure of the composite, homogenization theory can be applied to obtain the effective wave equations, in which the fluid and the solid coexist at ever point in the poroelastic material. M. A. Biot derived the governing equations for wave propagation in linear poroelastic composite materials in [4] and [5]. The former deals with the low-frequency regime where the friction between the viscous pore fluid and the elastic solid can be assumed to be linear proportional with the difference between the effective pore fluid velocity and the effective solid velocity by a real number $b$, which is independent of frequency $\omega$; this set of equation is referred to as the low-frequency Biot equation. When the frequency is higher than the critical frequency of the poroelastic material, $b$ will be frequencydependent; this is the subject of study in [5]. The exact form of $b$ as a function of frequency was derived in [5] for pore space with its micro-geometry being circular tubes. A more general expression was derived in the seminal paper [18] by Johnson, Koplik and Dashen (JKD), where causality argument was applied to derive the 'simplest' form of $b$ as a function of frequency. This frequency dependence of $b$ results in a time-convolution term in the time-domain poroelastic wave equations; the kernel in the time-convolution term is called the 'dynamic tortuosity' in the literature. The Biot-JKD equations refer to the Biot equations with $b$ being the JKD-tortuosity in (1).

In general, the dynamic tortuosity function is a tenor, which is related to the symmetric, positive definite dynamic permeability tensor of the poroelastic material $\mathbf{K}(\omega)$ by $T(\omega)=\frac{i \eta \phi}{\omega \rho_{f}} \mathbf{K}^{-1}(\omega)$. By the definition of dynamic tortuosity and dynamic permeability it is clear that their principal directions coincide. In the principal direction $x_{j}, j=1, \cdots, 3$ of $\mathbf{K}$, the JKD tortuosity is

$$
T_{j}^{J}(\omega)=\alpha_{\infty j}\left(1-\frac{\eta \phi}{i \omega \alpha_{\infty j} \rho_{f} K_{0 j}} \sqrt{1-i \frac{4 \alpha_{\infty j}^{2} K_{0 j}^{2} \rho_{f} \omega}{\eta \Lambda_{j}^{2} \phi^{2}}}\right), j=1,2,3
$$

\footnotetext{
${ }^{1}$ The work of MYO is partially sponsored by NSF-DMS-1413039

${ }^{2} \mathrm{HJW}$ is partially supported by Simons Foundation grant 355645
} 
with the tunable geometry-dependent constant $\Lambda_{j}$, the dynamic viscosity of pore fluid $\eta=\rho_{f} \nu$, the porosity $\phi$, the fluid density $\rho_{f}$, the static permeability $K_{j}$ and the infinite-frequency-tortuosity $\alpha_{\infty j}$; all of these parameters are positive real numbers. We refer to $T_{j}^{J}(\omega)$ as the JKD tortuosity function in the $j$-th direction..

The time-domain low-frequency Biot's equations have been numerically solved by many authors. However, for high-frequency Biot equations such as Biot-JKD, the time convolution term remains a challenge for numerical simulation. Masson and Pride [14] defined a time convolution product to discretize the fractional derivative. Lu and Hanyga [13] developed a new method to calculate the shifted fractional derivative without storing and integrating the entire velocity histories. Recent years, Chiavassa and Lombard et al. [8, 12] used an optimization procedure to approach the fractional derivative.

In this work, we will derive an equivalent system of the Biot-JKD equations without resorting to the fractional derivative technique. The advantage of this approach is that the new system of equations have the same structure as the low-frequency Biot equations but with more variables. Hence we refer to this system as the augmented Biot-JKD equations. A key step in this derivation is to utilize the Stieltjes function structure to compute from the given JKD tortuosity the coefficient of the additional terms.

The first-order formulation of the time-domain Biot equations consists of the strain-stress relations of the poroelastic materials and the equation of motions. The solid displacement $\mathbf{u}$, the pore fluid velocity relative to the solid $\boldsymbol{q}$ and the pore pressure $p$ are the unknowns to be solved. In terms of the solid displacement $\mathbf{u}$, we define the following variables

$\mathbf{v}:=\partial_{t} \mathbf{u}$ (solid velocity) $, \mathbf{w}:=\phi(\boldsymbol{U}-\mathbf{u})$ (fluid displacement relative to the solid) $, \boldsymbol{q}:=\partial_{\mathbf{t}} \mathbf{w}, \zeta:=-\nabla \cdot \mathbf{w}$

where $\phi$ is the porosity. Here $\boldsymbol{U}$ is the averaged fluid velocity over a representative volume element. The spatial coordinates $\left(x_{1}, x_{2}, x_{3}\right)$ are chosen to be aligned with the principal directions of the static permeability tensor $\boldsymbol{K}$ of the poroelastic material, which is know to be symmetric and positive definite. 6

Let $\epsilon_{i j}:=\frac{1}{2}\left(\frac{\partial u_{i}}{\partial x_{j}}+\frac{\partial u_{j}}{\partial x_{i}}\right)$ be the linear strain of the solid part, then the stress-strain relation is given by

$$
\left(\begin{array}{c}
\sigma_{11} \\
\sigma_{22} \\
\sigma_{33} \\
\sigma_{23} \\
\sigma_{13} \\
\sigma_{12} \\
p
\end{array}\right)=\left(\begin{array}{ccccccc}
c_{11}^{u} & c_{12}^{u} & c_{13}^{u} & c_{14}^{u} & c_{15}^{u} & c_{16}^{u} & M \alpha_{1} \\
c_{12}^{u} & c_{22}^{u} & c_{23}^{u} & c_{24}^{u} & c_{25}^{u} & c_{26}^{u} & M \alpha_{2} \\
c_{13}^{u} & c_{23}^{u} & c_{33}^{u} & c_{34}^{u} & c_{35}^{u} & c_{36}^{u} & M \alpha_{3} \\
c_{14}^{u} & c_{24}^{u} & c_{34}^{u} & c_{44}^{u} & c_{45}^{u} & c_{46}^{u} & M \alpha_{4} \\
c_{15}^{u} & c_{25}^{u} & c_{35}^{u} & c_{45}^{u} & c_{55}^{u} & c_{56}^{u} & M \alpha_{5} \\
c_{16}^{u} & c_{26}^{u} & c_{36}^{u} & c_{46}^{u} & c_{56}^{u} & c_{66}^{u} & M \alpha_{6} \\
M \alpha_{1} & M \alpha_{2} & M \alpha_{3} & M \alpha_{4} & M \alpha_{5} & M \alpha_{6} & M
\end{array}\right)\left(\begin{array}{c}
\epsilon_{11} \\
\epsilon_{22} \\
\epsilon_{33} \\
2 \epsilon_{23} \\
2 \epsilon_{13} \\
2 \epsilon_{12} \\
-\zeta
\end{array}\right)
$$

where $p$ is the pore pressure, $c_{i j}^{u}$ are the elastic constants of the undrained frame, which are related to the elastic constants $c_{i j}$ of the drained frame by $c_{i j}^{u}=c_{i j}+M a_{i} a_{j}, i, j=1, \ldots, 6$. In terms of the material bulk moduli $\kappa_{s}$ and $\kappa_{f}$ of the solid and the fluid, respectively, the fluid-solid coupling constants $a_{i}$ and $M$ are given by

$$
\begin{array}{r}
a_{i}:= \begin{cases}1-\frac{1}{3 \kappa_{s}} \sum_{k=1}^{3} c_{i k} & \text { for } i=1,2,3, \\
-\frac{1}{3 \kappa_{s}} \sum_{k=1}^{3} c_{k i} & \text { for } i=4,5,6,\end{cases} \\
M:=\frac{\kappa_{s}}{1-\bar{\kappa} / \kappa_{s}-\phi\left(1-\kappa_{s} / \kappa_{f}\right)}, \\
\bar{\kappa}:=\frac{c_{11}+c_{22}+c_{33}+2 c_{12}+2 c_{13}+2 c_{23}}{9} .
\end{array}
$$

The six equations of motion are as follows

$$
\begin{array}{r}
\sum_{k=1}^{3} \frac{\partial \sigma_{j k}}{\partial x_{k}}=\rho \frac{\partial v_{j}}{\partial t}+\rho_{f} \frac{\partial q_{j}}{\partial t}, t>0, \\
-\frac{\partial p}{\partial x_{j}}=\rho_{f} \frac{\partial v_{j}}{\partial t}+\left(\frac{\rho_{f}}{\phi}\right) \check{\alpha_{j} \star} \frac{\partial q_{j}}{\partial t}, t>0, j=1,2,3,
\end{array}
$$

where $\star$ denotes the time-convolution operator, $\rho_{f}$ and $\rho_{s}$ are the density of the pore fluid and of the solid, respectively, $\rho:=\rho_{s}(1-\phi)+\phi \rho_{f}$ and $\check{\alpha_{j}}$ is the inverse Laplace transform of the dynamic tortuosity $\alpha_{j}(\omega)$ 
with $\omega$ being the frequency. Here the one-sided Laplace transform of a function $f(t)$ is defined as

$$
\hat{f}(\omega):=\mathcal{L}[f](s=-i \omega):=\int_{0}^{\infty} f(t) e^{-s t} d t .
$$

As a special case of the Biot-JKD equations, the low frequency Biot's equation corresponds to

$$
\hat{\alpha_{j}}(t)=\alpha_{\infty j} \delta(t)+\frac{\eta \phi}{K_{0 j} \rho_{f}} H(t)
$$

where $\delta(t)$ is the Dirac function and $H(t)$ the Heaviside function, $\eta$ the dynamic viscosity of the pore fluid, $K_{0 j}$ the static permeability in the $x_{j}$ direction. This low-frequency tortuosity function corresponds to

$$
\alpha_{j}(\omega)=\alpha_{\infty j}+\frac{\eta \phi / K_{0 j} \rho_{f}}{-i \omega}
$$

In the Biot-JKD equation, we have $\alpha_{j}(\omega)=T_{j}^{J}(\omega)$.

According to Theorem 5.1 in [20], in the principal coordinates $\left\{x_{j}\right\}_{j=1}^{3}$ of the permeability tensor $\mathbf{K}$ and for $\omega$ such that $-\frac{i}{\omega} \in \mathbb{C} \backslash \theta_{1}$, the JKD dynamic tortuosity function has the following integral representation formula

$$
T_{j}^{J}(\omega)=a_{j}\left(\frac{i}{\omega}\right)+\int_{0}^{\theta_{1}} \frac{d \sigma_{j}(t)}{1-i \omega t}, a_{j}:=\frac{\eta \phi}{\rho_{f} K_{0 j}}, j=1,2,3
$$

where $0<\theta_{1}<\infty$ and the positive measure $d \sigma_{j}$ has a Dirac measure of strength $\alpha_{\infty j}$ sitting at $t=0$; this is to take into account the asymptotic behavior of dynamic tourturosity as frequency goes to $\infty$. This function is the analytic continuation of the usual dynamic tortuosity function in which $\omega \geq 0$. As a function of the new variable $s:=-i \omega, \omega \in \mathbb{C}$, the singularities of $(5)$ are included in the interval $\left(-\infty,-\frac{1}{\theta_{1}}\right)$ and a simple pole sitting at $s=0$. Therefore, if we define a new function for each $j=1,2,3$

$$
D_{j}^{J}(s):=T_{j}^{J}(\omega)-\frac{i a_{j}}{\omega}=\int_{0}^{\theta_{1}} \frac{d \sigma_{j}(t)}{1+s t},
$$

then $D_{j}^{J}(s)$ is analytic in $\mathbb{C} \backslash\left(-\infty,-\frac{1}{\theta_{1}}\right)$ on the s-plane. This type of functions are closely related to the well-known Stieltjes functions. The first approach we propose in this paper is based on the fact [16] that a Stieltjes function can be well approximated by its Padé approximant whose poles are all simple. The other approach proposed here for computing the pole-residue approximation of the dynamic tortuosity function is based on the result in [1].

We note that

$$
D_{j}^{J}(s)=\alpha_{\infty j}\left(1+\frac{\eta \phi}{s \alpha_{\infty j} \rho_{f} K_{0 j}} \sqrt{1+s \frac{4 \alpha_{\infty j}^{2} K_{0 j}^{2} \rho_{f}}{\eta \Lambda_{j}^{2} \phi^{2}}}\right)-\frac{a_{j}}{s}=: \alpha^{J}(s)-\frac{a_{j}}{s}, j=1,2,3
$$

is analytic away from the branch cut on $\left[0, C_{1}\right]$ along the real axis, where $C_{1}:=\frac{4 \alpha_{\infty j}^{2} K_{0 j}^{2}}{\nu \phi^{2} \Lambda_{j}^{2}}$. Therefore

$$
D_{j}^{J}(s)=\int_{0}^{C_{1}} \frac{d \sigma_{j}^{J}(t)}{1+s t} \approx \alpha_{\infty}+\sum_{k=1}^{M} \frac{r_{k}^{j}}{s-p_{k}^{j}} \text { for } s \in \mathbb{C} \backslash\left(-\infty,-\frac{1}{C_{1}}\right], j=1,2,3 .
$$

with $r_{k}^{j}>0, p_{k}^{j}<-\frac{1}{C_{1}}<0, j=1,2,3, k=1, \ldots, M$, that can be computed from dynamic permeability data $K_{j}(\omega)$ evaluated at $M$ different frequencies in the frequency content of the initial waves. The special choice of $s=-i \omega, \omega \in \mathbb{R}$ in 7 provides a pole-residue approximation of $T_{j}^{J}(\omega), j=1,2,3$. 
Applying Laplace transform to the convolution term in (4) with JKD tortuosity, i.e. $\alpha=\alpha^{J}$, (see eg. Theorem 9.2.7 in [10])

$$
\begin{aligned}
\mathcal{L}\left[\tilde{\alpha^{J}} \star \frac{\partial q_{j}}{\partial t}\right](s) & =\alpha^{J}(s)\left(s \hat{q_{j}}\right) \\
& =\left(D^{J}(s)+\frac{a}{s}\right)\left(s \hat{q_{j}}\right) \\
& \approx\left(\alpha_{\infty}+\sum_{k=1}^{M} \frac{r_{k}}{s-p_{k}}+\frac{a}{s}\right)\left(s \hat{q_{j}}\right) \\
& \approx \alpha_{\infty} s \hat{q_{j}}+\sum_{k=1}^{M} \frac{r_{k}}{s-p_{k}}\left(s \hat{q_{j}}\right)+a \hat{q_{j}} \\
& =\alpha_{\infty} s \hat{q_{j}}+\left(a+\sum_{k=1}^{M} r_{k}\right) \hat{q}_{j}+\sum_{k=1}^{M} r_{k} p_{k} \frac{\hat{q_{j}}}{s-p_{k}} .
\end{aligned}
$$

Notice that

$$
s \hat{q_{j}}=\mathcal{L}\left[\partial_{t} q^{j}\right]+q_{j}(0) .
$$

Furthermore, for each of the terms in the sum, since all the singularities $p_{k}$ are restricted to the left of $s=-\frac{1}{C_{1}}$, the inverse Laplace transform can be performed by integrating along the imaginary axis for $t>0$, i.e.,

$$
\mathcal{L}^{-1}\left[\frac{1}{s-p_{k}}\right](t)=\frac{1}{2 \pi i} \int_{-i \infty}^{i \infty} \frac{1}{\zeta-p_{k}} e^{\zeta t} d \zeta=r_{k} e^{p_{k} t}, t>0 .
$$

This integral is evaluated by integrating along $[-R i, R i] \cup\left\{s=R e^{i \theta} \mid \pi / 2<\theta<3 \pi / 2\right\}$ and applying the residue theorem and letting $R \rightarrow \infty$. As a result, we have for $t>0$

$$
\begin{aligned}
& \left(\check{\alpha^{J}} \star \frac{\partial q_{j}}{\partial t}\right)(\boldsymbol{x}, t):=\int_{0}^{t} \tilde{\alpha}^{J}(\tau) \frac{\partial q_{j}}{\partial t}(\boldsymbol{x}, t-\tau) d \tau \\
& \approx \alpha_{\infty}\left(\frac{\partial q_{j}}{\partial t}\right)+\left(a+\sum_{k=1}^{M} r_{k}\right) q_{j}-\sum_{k=1}^{M} r_{k}\left(-p_{k}\right) e^{p_{k} t} \star q_{j} .
\end{aligned}
$$

Applying a strategy similar to those in the literature [9], we define the auxiliary variables $\Theta_{k}, k=$ $1, \ldots, M$ such that

$$
\Theta_{k}^{x_{j}}(\boldsymbol{x}, t):=\left(-p_{k}\right) e^{p_{k} t} \star q_{j} .
$$

It can be easily checked that $\Theta_{k}, k=1, \ldots, M$, satisfies the following equation:

$$
\partial_{t} \Theta_{k}^{x_{j}}(\boldsymbol{x}, t)=p_{k} \Theta_{k}^{x_{j}}(\boldsymbol{x}, t)-p_{k} q_{j}(\boldsymbol{x}, t)
$$

We assume $\mathbf{q}(0)=0$ for simplicity. For an anisotropic media, each principal direction $x_{j}, j=1,2,3$, has a different tortuosity function $\alpha_{j}$. We label the corresponding poles and residue as $p_{k}^{x_{j}}$ and $r_{k}^{x_{j}}$ and modify (16) accordingly. Replacing the convolution terms in (4) with the equations of $\Theta_{k}^{x_{j}}$, we obtain the following system that has no explicit memory terms:

$$
\left\{\begin{aligned}
\sum_{k=1}^{3} \frac{\partial \sigma_{j k}}{\partial x_{k}} & =\rho \frac{\partial v_{j}}{\partial t}+\rho_{f} \frac{\partial q_{j}}{\partial t}, t>0, \\
\partial_{t} \Theta_{k}^{x_{j}}(\boldsymbol{x}, t) & =p_{k} \Theta_{k}^{x_{j}}(\boldsymbol{x}, t)-p_{k} q_{j}(\boldsymbol{x}, t), j=1,2,3, \\
-\frac{\partial p}{\partial x_{j}} & =\rho_{f} \frac{\partial v_{j}}{\partial t}+\left(\frac{\rho_{f} \alpha_{\infty j}}{\phi}\right) \frac{\partial q_{j}}{\partial t}+\left(\frac{\eta}{K_{0 j}}+\frac{\rho_{f}}{\phi} \sum_{k=1}^{M} r_{k}\right) q_{j} \\
& -\left(\frac{\rho_{f}}{\phi}\right) \sum_{k=1}^{M} r_{k} \Theta_{k}^{x_{j}}, t>0, j=1,2,3 .
\end{aligned}\right.
$$

We refer to this system as the augmented system of Biot-JKD equations in the principal directions of the permeability tensor $\mathbf{K}$. 


\section{Numerical scheme for computing $r_{k}$ and $p_{k}$}

Since the function $D^{J}$ results from subtracting the pole of $T^{J}$ at $s=0$, it has a removable singularity at $s=0$ and is analytic away from its branch-cut located at $\left(-\infty,-1 / C_{1}\right]$. Both a Approachpproachs presented here are based on the fact that $D^{J}(s)$ is a Stieltjes function.

The problem to be solved is formulated as follows. Given the data of $D^{J}$ at distinct values of $s=$ $s_{1}, \ldots, s_{M}$, construct the pole-residue approximation of $D^{J}$ such that

$$
D^{J}(s) \approx D_{\text {est }}^{J}(s):=\alpha_{\infty}+\sum_{k=1}^{M} \frac{r_{k}}{s-p_{k}} \text { for } s \in\left[s_{1}, s_{M}\right] \text { and } r_{k}>0, p_{k}<0, \forall k=1, \ldots, M .
$$

\subsection{Rational function approximation and partial fraction decomposition}

This approximation takes into account the asymptotic behavior $\lim _{s \rightarrow \infty}=\alpha_{\infty}$ and hence can be considered as an improved version of the reconstruction Approach for tortuosity in [20], which does not interpolate at infinity. In this paper, we also take into account the asymptotic behaviors of $D(s)$. Note that

$$
\lim _{\omega \rightarrow 0^{+}} D(s=-i \omega)=\alpha_{\infty}+2\left(\frac{\alpha_{\infty}}{\Lambda}\right)^{2} \frac{K_{0}}{\phi}, \quad \quad \lim _{\omega \rightarrow \infty} D(s=-i \omega)=\alpha_{\infty}
$$

By a theorem in [16], we know that the poles in the Padé approximant of $D^{J}(s)$ have to be contained in $\left(-\infty,-1 / C_{1}\right]$ and are all simple with positive weight (residue), this implies that the constant term in the denominator in the Padé approximant can be normalized to one. According to the aforementioned theorem, if $\left(s, D^{J}(s)\right)$ is an interpolation point with $\operatorname{Im}(s) \neq 0$, then $\left(\bar{s}, D^{J}(\bar{s})\right)$ must also be an interpolation point, where - represents the complex conjugate. From the integral representation formula (IRF), we know that $D^{J}\left(\overline{s_{k}}\right)=\overline{D^{J}\left(s_{k}\right)}$.

Hence, the following approximation problem is considered: Given $M$ data points $D^{J}\left(s_{k}=-i \omega_{k}\right) \in \mathbb{C}$, $k=1, \ldots, M$, find $\boldsymbol{x}:=\left(a_{0}, \cdots, a_{M-1}, b_{1}, \cdots, b_{M}\right)^{t}$ such that

$$
(S)\left\{\begin{array}{l}
D^{J}\left(s_{k}\right)-\alpha_{\infty}=\frac{a_{0}+a_{1} s_{k}+\cdots+a_{M-1} s_{k}^{M-1}}{1+b_{1} s_{k}+\cdots+b_{M} s_{k}^{M}}, k=1, \ldots, M, \\
\overline{D^{J}\left(s_{k}\right)}-\alpha_{\infty}=\frac{a_{0}+a_{1} \overline{s_{k}}+\cdots+a_{M-1} \bar{s}_{k} M-1}{1+b_{1} \overline{s_{k}}+\cdots+b_{M} \overline{s_{k}} M}, k=1, \ldots, M,
\end{array}\right.
$$

where $\omega_{k}, k=1, \ldots, M$, are distinct positive numbers. However, with a closer look, this system of equations is equivalent to the one by enforcing the condition $\boldsymbol{x} \in \mathbb{R}^{2 M}$ to the first half of $(S)$. To be more specific, we define

$$
\begin{aligned}
A & :=\left(\begin{array}{ccccccccc}
1 & s_{1} & s_{1}^{2} & \cdots & s_{1}^{M-1} & -D^{J}\left(s_{1}\right) s_{1} & -D^{J}\left(s_{1}\right) s_{1}^{2} & \cdots & -D^{J}\left(s_{1}\right) s_{1}^{M} \\
1 & s_{2} & s_{2}^{2} & \cdots & s_{2}^{M-1} & -D^{J}\left(s_{2}\right) s_{2} & -D^{J}\left(s_{2}\right) s_{2}^{2} & \cdots & -D^{J}\left(s_{2}\right) s_{2}^{M} \\
\vdots & \vdots & \vdots & & \vdots & \vdots & \vdots & & \vdots \\
1 & s_{M} & s_{M}^{2} & \cdots & s_{M}^{M-1} & -D^{J}\left(s_{M}\right) s_{M} & -D^{J}\left(s_{M}\right) s_{M}^{2} & \cdots & -D^{J}\left(s_{M}\right) s_{M}^{M}
\end{array}\right) \in \mathbb{C}^{M \times 2 M}, \\
\boldsymbol{d} & :=\left(D^{J}\left(s_{1}\right)-\alpha_{\infty}, D^{J}\left(s_{2}\right)-\alpha_{\infty}, \cdots D^{J}\left(s_{M}\right)-\alpha_{\infty}\right)^{t} \in \mathbb{C}^{M} \\
\boldsymbol{x} & :=\left(a_{0}, \cdots, a_{M-1}, b_{1}, \cdots, b_{M}\right)^{t} \in \mathbb{R}^{M} .
\end{aligned}
$$

Then the system to be solved is

$$
\left(\begin{array}{c}
\operatorname{Re}(A) \\
\operatorname{Im}(A)
\end{array}\right) \boldsymbol{x}=\left(\begin{array}{c}
\operatorname{Re}(\boldsymbol{d}) \\
\operatorname{Im}(\boldsymbol{d})
\end{array}\right)
$$

where $\operatorname{Re}()$ and $\operatorname{Im}()$ denote the real part and the imaginary part, respectively. After solving for $\boldsymbol{x}$, the poles and residues are then obtained by the partial fraction decomposition of the Padé approximant, i.e.

$$
\frac{a_{0}+a_{1} s+\cdots+a_{M} s^{M-1}}{1+b_{1} s+\cdots+b_{M} s^{M}}=\sum_{j=1}^{M} \frac{r_{j}}{s-p_{j}} .
$$




\subsection{Two-sided residue interpolation in the Stieltjes class}

The second approach is based on the following theorem that can be considered as a special case of what is proved in [1]. The advantage of this method is that it explicitly identifies the poles $p_{k}, k=1, \ldots, M$ as the generalized eigenvalues of matrices constructed from the data. We note that the interpolation problem below also appears in the recent paper [2], where the main focus is model reduction.

Let $\mathbb{C}^{+}:=\{z \in \mathbb{C}: \operatorname{Im}(z)>0\}$. Given $M$ interpolation data $\left(z_{i}, u_{i}, v_{i}\right) \in \mathbb{C}^{+} \times \mathbb{C}^{p \times q} \times \mathbb{C}^{p \times q}$, we seek a $p \times p$ matrix valued function $F(z)$ of the form

$$
F(z)=\int_{0}^{\infty} \frac{d \mu(t)}{t-z}, \text { where } \mu \text { is a positive } p \times p \text { matrix -valued measure }
$$

such that

$$
F\left(z_{i}\right) u_{i}=v_{i}, i=1, \ldots, M .
$$

Theorem 2.1. If there exists a solution $F(z)$ described as above, then the Hermitian matrices $S_{1}$ and $S_{2}$ defined via

$$
\left(S_{1}\right)_{i j}=\frac{u_{i}^{*} v_{j}-v_{i}^{*} u_{j}}{z_{j}-\overline{z_{i}}},\left(S_{2}\right)_{i j}:=\frac{z_{j} u_{i}^{*} v_{j}-\overline{z_{i}} v_{i}^{*} u_{j}}{z_{j}-\overline{z_{i}}}, i, j=1, \ldots, M,
$$

are positive semidefinite. Conversely, if $S_{1}$ is positive definite and $S_{2}$ is positive semidefinite, then

$$
F(z):=-C_{+}\left(z S_{1}-S_{1} A-C_{+}^{*} C_{-}\right)^{-1} C_{+}^{*}=C_{+}\left(S_{2}-z S_{1}\right)^{-1} C_{+}^{*}
$$

is a solution to the interpolation problem. Here

$$
C_{-}:=\left(\begin{array}{lll}
u_{1} & \cdots & u_{M}
\end{array}\right), C_{+}:=\left(\begin{array}{lll}
v_{1} & \cdots & v_{M}
\end{array}\right), A:=\operatorname{diag}\left(z_{i} I_{q}\right)_{i=1}^{M},
$$

and $I_{q}$ is the identity matrix of dimension $q$.

Proof. Suppose 26] and 27] are true. Then we have

$$
u_{i}^{*} v_{j}-v_{i}^{*} u_{j}=u_{i}^{*}\left(F\left(z_{j}\right)-F\left(z_{i}\right)^{*}\right) u_{j}=\left(z_{j}-\overline{z_{i}}\right) u_{i}^{*}\left(\int_{0}^{\infty} \frac{d \mu(t)}{\left(t-z_{j}\right)\left(t-\overline{z_{i}}\right)}\right) u_{j} .
$$

Thus

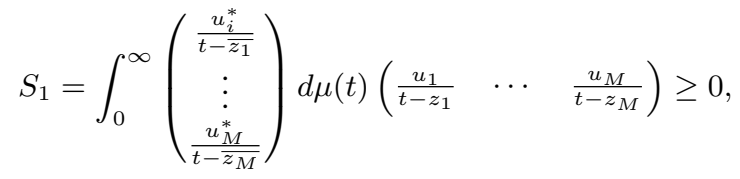

$$
\begin{aligned}
& S_{2}=\int_{0}^{\infty}\left(\begin{array}{c}
\frac{u_{i}^{*}}{t-\bar{z}_{1}} \\
\vdots \\
u_{M}^{*}
\end{array}\right) t d \mu(t)\left(\begin{array}{lll}
\frac{u_{1}}{t-z_{1}} & \cdots & \frac{u_{M}}{t-z_{M}}
\end{array}\right) \geq 0 .
\end{aligned}
$$

Conversely, suppose $S_{1}>0$ and $S_{2} \geq 0$. Notice that

$$
\left\{\begin{array}{l}
A^{*} S_{1}-S_{1} A=C_{+}^{*} C_{-}-C_{-}^{*} C_{+}, \\
A^{*} S_{2}-S_{2} A=A^{*} C_{+}^{*} C_{-}-C_{-}^{*} C_{+} A .
\end{array}\right.
$$

These equations uniquely determine $S_{1}$ and $S_{2}$ as the spectra of $A$ and $A^{*}$ do not overlap. Observe that if $S_{1}$ satisfies 30, then $S_{2}:=S_{1} A+C_{+}^{*} C_{-}$is the solution of 31. Therefore, we have

$$
S_{2}=S_{1} A+C_{+}^{*} C_{-} .
$$

Note that $S_{2}-z S_{1}=S_{1}^{\frac{1}{2}}\left(S_{1}^{-\frac{1}{2}} S_{2} S_{1}^{-\frac{1}{2}}-z\right) S_{1}^{\frac{1}{2}}$. Since $S_{1}^{-\frac{1}{2}} S_{2} S_{1}^{-\frac{1}{2}}$ has eigen values in $[0, \infty),\left(S_{1}^{-\frac{1}{2}} S_{2} S_{1}^{-\frac{1}{2}}-z\right)$ is invertible for $z \notin[0, \infty)$. Let $(X, D)$ be the eigen decomposition such that

$$
S_{1}^{-\frac{1}{2}} S_{2} S_{1}^{-\frac{1}{2}}=X D X^{*} \text { with } X=\left(\begin{array}{lll}
\boldsymbol{x}_{1} & \cdots & \boldsymbol{x}_{q M}
\end{array}\right), D=\operatorname{diag}\left(d_{j}\right)_{j=1}^{q M} .
$$


Then we have for $z \notin[0, \infty)$

$$
F(z)=\sum_{j=1}^{q M}\left(\frac{1}{d_{j}-z}\right) C_{+} S_{1}^{-\frac{1}{2}} \boldsymbol{x}_{j} \boldsymbol{x}_{j}^{*} S_{1}^{-\frac{1}{2}} C_{+}^{*},
$$

and thus $F(z)$ has the required form with $d \mu$ being a atomic measure supported on $d_{1}, \ldots, d_{q M}$.

Furthermore, letting $\boldsymbol{e}_{1}, \ldots, \boldsymbol{e}_{M}$ be the standard basis vectors of $\mathbb{R}^{M}$, we have for $i=1, \ldots, M$,

$$
\left(z_{i} S_{1}-S_{1} A-C_{+}^{*} C_{-}\right)\left(\boldsymbol{e}_{i} \otimes I_{q}\right)=S_{1}\left(z_{i}-I-A\right)\left(\boldsymbol{e}_{i} \otimes I_{q}\right)-C_{+}^{*} C_{-}\left(\boldsymbol{e}_{i} \otimes I_{q}\right)=0-C_{+}^{*} u_{i}=-C_{+}^{*} u_{i} .
$$

Thus

$$
F\left(z_{i}\right) u_{i}=-C_{+}\left(z_{i} S_{1}-S_{1} A-C_{+}^{*} C_{-}\right)^{-1} C_{+}^{*} u_{i}=-C_{+}\left(-\boldsymbol{e}_{i} \otimes I_{q}\right)=v_{i}
$$

To apply this theorem to our problem, we first note that if we identify $z$ in Theorem 42 with $-\frac{1}{s}$, then the IRF for $D_{j}^{J}(s)$ in (7), denoted by $D^{J}$ for simplicity, can be written as

and

$$
D^{J}(s)=(-z) \int_{0}^{\Theta_{1}} \frac{d \sigma^{J}}{t-z}
$$

$$
D^{J}(s)-\alpha_{\infty}=(-z)\left(\int_{0}^{\Theta_{1}} \frac{d \sigma^{J}(t)}{t-z}-\frac{\alpha_{\infty}}{-z}\right)=(-z)\left(\int_{0}^{\Theta_{1}} \frac{d \sigma^{J}(t)}{t-z}-\int_{0}^{\Theta_{1}} \frac{\alpha_{\infty} \sigma(t)}{t-z}\right),
$$

where $\sigma(t)$ is a Dirac measure at $t=0$. Since $\sigma^{J}$ has a Dirac measure of strength $\alpha_{\infty}$, the function inside the parentheses in 33 is a Stieltjes function, which we denote by $F_{n e w}(z)$, i.e.

$$
D^{J}(s)-\alpha_{\infty}=(-z) F_{n e w}(z)
$$

What we would like to harvest is the pole-residue approximation of $D(s)-\alpha_{\infty}$. To avoid truncation error, we rewrite all the formulas in Theorem 42 in terms of variable $s=-\frac{1}{z}$ as follows.

$$
\begin{array}{r}
s_{i}=-\frac{1}{z_{i}}, u_{i}=\frac{1}{s_{i}}, \\
v_{i}=D\left(s_{i}\right)-\alpha_{\infty}, i=1 \ldots M \\
\left(S_{1}\right)_{i j}=\frac{-s_{j} D\left(s_{j}\right)+s_{i}^{*} D^{*}\left(s_{i}\right)}{s_{i}^{*}-s_{j}}, \\
\left(S_{2}\right)_{i j}=\frac{-D\left(s_{j}\right)+D^{*}\left(s_{i}\right)}{s_{j}-s_{i}^{*}} .
\end{array}
$$

Consequently, we have the following representation for $D(s)$

$$
D^{J}(s) \equiv \alpha_{\infty}+\left(\frac{1}{s}\right) F_{n e w}\left(-\frac{1}{s}\right)=\alpha_{\infty}+\sum_{j=1}^{q M}\left(\frac{1}{s d_{j}+1}\right) C_{+} S_{1}^{-\frac{1}{2}} \boldsymbol{x}_{j} \boldsymbol{x}_{j}^{*} S_{1}^{-\frac{1}{2}} C_{+}^{*} .
$$

With the generalized eigenvalues $[\mathbf{V}, \mathbf{L}]:=\operatorname{eig}\left(S_{1}, S_{2}\right)$, where $\mathbf{V}$ is the matrix of generalized vectors and $\mathbf{L}$ the diagonal matrix of generalized eigenvalues such that

$$
S_{1} \mathbf{V}=S_{2} \mathbf{V L}
$$

and taking into account of the simultaneous diagonalization property

$$
\begin{aligned}
& \mathbf{V}^{*} \mathbf{S}_{1} \mathbf{V}=\mathbf{L} \\
& \mathbf{V}^{*} \mathbf{S}_{2} \mathbf{V}=\mathbf{I}
\end{aligned}
$$

we have

$$
D^{J}(s)=\alpha_{\infty}+\sum_{k=1}^{N} \frac{C_{+} \mathbf{V}(:, k) \mathbf{V}(:, k)^{*} C_{+}^{*}}{s+L(k, k)} .
$$

The poles $\vartheta_{k}$ and residues $r_{k}$ are given by

$$
\begin{aligned}
p_{k} & =-L(k, k) \\
r_{k} & =C_{+} \mathbf{V}(:, k) \mathbf{V}(:, k)^{*} C_{+}^{*}
\end{aligned}
$$




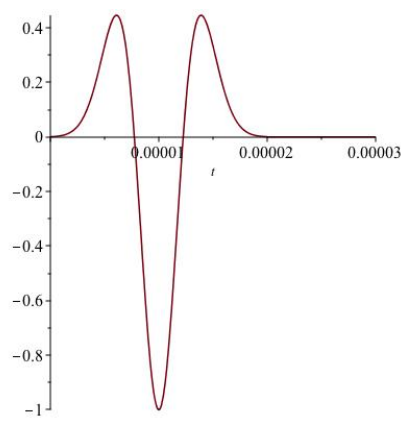

Figure 1: Ricker wavelet $g(t)$

\section{Numerical Examples}

In this section, we apply both approachs in Section 2 to the examples of cancellous bone (S1) studied in [17], [11] and the epoxy-glass mixture (S2 and S3) and the sandstone (S4 and S5) examples studied in [7]. From prior results, it is known that wider range the frequency is, the more ill-conditioned the corresponding matrices will be. We focus on the test case in [7], which apply the fractional derivate approach to deal with the memory term. In this case, time profile of the source term, denoted by $g(t)$ is a Ricker signal of central frequency $f_{0}=10^{5} \mathrm{~s}^{-1}$ and time-shift $t_{0}=1 / f_{0}$, i.e.

$$
g(t)= \begin{cases}\left(2 \pi^{2} f_{0}^{2}\left(t-t_{0}\right)^{2}-1\right) \exp \left(-\pi^{2} f_{0}^{2}\left(t-t_{0}\right)^{2}\right), & \text { if } 0 \leq t \leq 2 t_{0} \\ 0, & \text { otherwise }\end{cases}
$$

See Figure 1

The spectrum content of $g(t)$ is visualized by its Fourier transform $\mathcal{F}\{g\}(\omega)$, see Figure $2 \mathrm{a}$ and $2 \mathrm{~b}$ Since the real part and the imaginary part is symmetric and anti-symmetric with respect to $\omega=0$, respectively, we only plot the $\omega \geq 0$ part of the graphs. Based on Figure $2 \mathrm{a}$ and Figure $2 \mathrm{~b}$, we choose the frequency range in our numerical simulation to be from $10^{-3} \mathrm{~Hz}$ to $2 \times 10^{6} \mathrm{~Hz}$.

We consider first the equal spaced sample points. Similar to what was reported in [20], the relative error peaked near low frequency. This is due to fact that in general, the function $D(s=-i \omega)$ varies the most near the lower end of $\omega$. This observation leads to the log-distributed grid points, which in general performs better in terms of maximum relative errors but with more ill-conditioned matrices. For both the equally spaced and the log-spaced grids point, ill-conditioned matrices are involved. The ill-conditioning nature of the matrices $A$ in Approach 1 and $S_{1}, S_{2}$ in Approach 2, together with the fact there is no obvious preconditioner available for these matrices, we resort to the multiprecision package Advanpix [15] for directly solving (24) and the subsequent partial fraction decomposition involved in Approach 1 and for solving the generalized eigenvalue problem (39). These real-valued poles and residues are then converted to double precision before we evaluate the relative errors

$$
\text { rel_err }(s):=\frac{\left|D^{J}(s)-D_{\text {est }}^{J}(s)\right|}{\left|D^{J}(s)\right|},
$$


Table 1: Biot-JKD parameters

\begin{tabular}{|c|c|c|c|c|c|c|}
\hline & & S1 & S2 & S3 & S4 & S5 \\
\hline$\rho_{f}\left(K g \cdot m^{-3}\right)$ & pore fluid density & 1000 & 1040 & 1040 & 1040 & 1040 \\
\hline$\phi$ (dimensionless) & porosity & 0.8 & 0.2 & 0.2 & 0.2 & 0.2 \\
\hline$\alpha_{\infty}$ (dimensionless) & infinite-frequency tortuosity & 1.1 & 3.6 & 2.0 & 2.0 & 3.6 \\
\hline$K_{0}\left(m^{2}\right)$ & static permeability & $3 \mathrm{e}-8$ & $1 \mathrm{e}-13$ & $6 \mathrm{e}-13$ & $6 \mathrm{e}-13$ & $1 \mathrm{e}-13$ \\
\hline$\nu\left(m^{2} \cdot s^{-1}\right)$ & kinematic viscosity of pore fluid & $1 \mathrm{e}-3 / \rho_{f}$ & $1 \mathrm{e}-3 / \rho_{f}$ & $1 \mathrm{e}-3 / \rho_{f}$ & $1 \mathrm{e}-3 / \rho_{f}$ & $1 \mathrm{e}-3 / \rho_{f}$ \\
\hline$\Lambda(m)$ & structure constant & $2.454 \mathrm{e}-5$ & $3.790 \mathrm{e}-6$ & $6.930 \mathrm{e}-6$ & $2.190 \mathrm{e}-7$ & $1.20 \mathrm{e}-7$ \\
\hline
\end{tabular}

where the pole-residue approximation function $D_{\text {est }}^{J}$ is defined in (21). We set the number of significant digits in Advanpix to be 90, which is much higher than the 15 decimal digits a 64-bit double-precision floating point format can represent.

The relative error rel_err with $M=10$ for all the 5 media listed in Table 1 is plotted in Figure 3a to Figure $3 \mathrm{e}$ The results by using equally space grids are in color blue while those by using log-distributed ones are in color red.

Among all the 5 media listed in Table 1. the cancellous bone S1 and the sandstones S4 and S5 are the most difficult one to approximate in the sense that it requires the largest $M$ for achieving the same level of accuracy as for other media. The dynamic tortuosity functions of S1, S4 and S5 have large variation near low frequency and hence can be approximated much better when log-distributed grids are applied in the approximations. See Figure $10 \mathrm{a}$ and Figure $10 \mathrm{~b}$

In Tables 2 to 6 , we list the condition numbers for both of the equally-spaced grid points and the $\log$-distributed one. As can be seen, the condition numbers for matrices involved in Approach 1 with logdistributed grid points worsen very rapidly with the increase of $M$ and the rescaling of volumes of $A$ is not effective when compared with the equally spaced case. In Figures 4 to 8 , where the poles and residues for $M=14$ computed with different combinations of methods are plotted in log-log scale, we see that Approach 1 and 2 indeed give numerically identical results for all these 5 test media when the significant digits in the calculations are set much higher than the $\log _{10}$-scale of the condition numbers involved. The calculation is carried out by using 140 significant digits and it takes about 5 seconds with a single processor MacBook Pro.

Table 2: $\log _{10}$-Condition numbers of the matrices for material S1

\begin{tabular}{|c|c|c|c|c|}
\hline & $\mathrm{M}=8$ & $\mathrm{M}=8$ & $\mathrm{M}=14$ & $\mathrm{M}=14$ \\
\hline & Equally spaced & log-spaced & Equally spaced & log-spaced \\
\hline$A$ & 48.9750 & 49.2328 & 86.7853 & 87.4283 \\
\hline$B$ & 10.0354 & 31.4021 & 15.2592 & 57.8602 \\
\hline$S_{1}$ & 13.3237 & 4.3014 & 23.7936 & 5.8767 \\
\hline$S_{2}$ & 9.9319 & 10.8471 & 30.3962 & 11.9522 \\
\hline
\end{tabular}

In Figure 9. the relative error rel_err for approximations by using equally spaced grid and by logdistributed grids are presented. As can be see from Figure 10a, the peak of error near the lower end of the frequency range is due to the fact that the function being approximated needs more grid points there to resolve the variation. This is achieved by using the log-distributed grids. In Figure 10a and Figure $10 \mathrm{~b}$ we plot $D^{J}$ and its pole-residue approximation $D_{\text {est }}^{J}$ to visualize the performance. Figure 10 a corresponds to the equally spaced grids while Figure $10 \mathrm{~b}$ to the log-distributed one. In both figures, these two functions are almost indiscernible except the imaginary parts in Figure 10a near the lower end of frequency where rel_err peaks; both the colors black (imaginary part of $D^{J}$ ) and green (imaginary part of $D_{\text {est }}^{J}$ ) can be seen there. 
Table 3: $\log _{10}$-Condition numbers of the matrices for material S2

\begin{tabular}{|c|c|c|c|c|}
\hline & $\mathrm{M}=8$ & $\mathrm{M}=8$ & $\mathrm{M}=14$ & $\mathrm{M}=14$ \\
\hline & Equally spaced & log-spaced & Equally spaced & log-spaced \\
\hline$A$ & 50.3092 & 51.1536 & 88.1203 & 90.5197 \\
\hline$B$ & 20.1795 & 64.8793 & 31.3151 & 120.2955 \\
\hline$S_{1}$ & 15.1483 & 55.9887 & 29.1822 & 110.3533 \\
\hline$S_{2}$ & 15.1657 & 55.9766 & 29.1998 & 110.3456 \\
\hline
\end{tabular}

Table 4: $\log _{10}$-Condition numbers of the matrices for material S3

\begin{tabular}{|c|c|c|c|c|}
\hline & $\mathrm{M}=8$ & $\mathrm{M}=8$ & $\mathrm{M}=14$ & $\mathrm{M}=14$ \\
\hline & Equally spaced & log-spaced & Equally spaced & log-spaced \\
\hline$A$ & 49.9052 & 50.8287 & 87.7160 & 90.1957 \\
\hline$B$ & 16.5867 & 59.7475 & 24.6405 & 110.6680 \\
\hline$S_{1}$ & 12.3521 & 49.2816 & 24.0606 & 97.5660 \\
\hline$S_{2}$ & 12.5097 & 49.2655 & 24.2122 & 97.5514 \\
\hline
\end{tabular}

\section{Conclusions}

In this paper, we utilize the Stieltjes function structure of the JKD dynamic tortuosity to derive an augmented system of Biot-JKD equations [18 - 20 that approximates the solution of the original Biot-JKD equations (3)-(4). Asymptotic behavior of the tortuosity function as $\omega \rightarrow \infty$ is enforced analytically before the numerical interpolation carried out by Approach 1 and Approach 2. Due to the nature of the tortuosity functions of S1, S4 and S5, log-distributed interpolation points generally perform better than the equally distributed ones. We tested our approachs on 5 sets of poroelastic parameters obtaining from the existing literature and interpolated the JKD dynamic tortuosity equation to high accuracy through a frequency range that spans 9 orders of magnitude from $10^{-3}$ to $2 \times 10^{6}$. The extremely ill-conditioned matrices are dealt with by using a multiprecision package Advanpix in which we set the significant digits of floating numbers to be 140. It turns out approachs 1 and 2 give numerically identical results for all the test cases when the significant digits are set much higher than the $\log _{10}$-scale of the condition numbers involved. We think the exact link between these two approachs can be derived through the Barycentric forms for rational approximations [3], which in term provides an approach that can adapt the choice of grid points based on the data points so the Lebesgue constant is minimized [19]. This will be explored in a later work.

Acknowledgments. We wish to thank Joe Ball, Daniel Alpay, Marc van Barel, Thanos Antoulas, Sanda Lefteriu, and Cosmin Ionita for their helpful suggestions. 
Table 5: $\log _{10}$-Condition numbers of the matrices for material S4

\begin{tabular}{|c|c|c|c|c|}
\hline & $\mathrm{M}=8$ & $\mathrm{M}=8$ & $\mathrm{M}=14$ & $\mathrm{M}=14$ \\
\hline & Equally spaced & log-spaced & Equally spaced & log-spaced \\
\hline$A$ & 50.8209 & 51.4408 & 88.6312 & 89.5011 \\
\hline$B$ & 11.8964 & 41.4130 & 16.7579 & 76.4563 \\
\hline$S_{1}$ & 11.5699 & 18.8133 & 22.0503 & 38.7220 \\
\hline$S_{2}$ & 14.6234 & 18.7833 & 25.0888 & 38.6911 \\
\hline
\end{tabular}

Table 6: $\log _{10}$-Condition numbers of the matrices for material S5

\begin{tabular}{|c|c|c|c|c|}
\hline & $\mathrm{M}=8$ & $\mathrm{M}=8$ & $\mathrm{M}=14$ & $\mathrm{M}=14$ \\
\hline & Equally spaced & log-spaced & Equally spaced & log-spaced \\
\hline$A$ & 51.0851 & 51.8269 & 88.8954 & 89.9913 \\
\hline$B$ & 12.2279 & 43.8165 & 17.1587 & 80.9135 \\
\hline$S_{1}$ & 11.3409 & 23.0730 & 21.8547 & 47.0251 \\
\hline$S_{2}$ & 13.8633 & 23.0477 & 24.3312 & 46.9971 \\
\hline
\end{tabular}

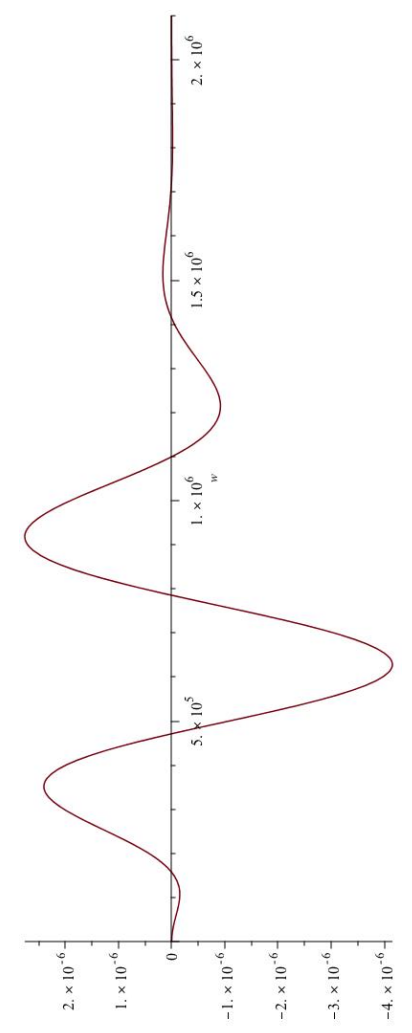

(a) Real part of $\mathcal{F}\{g\}(\omega)$

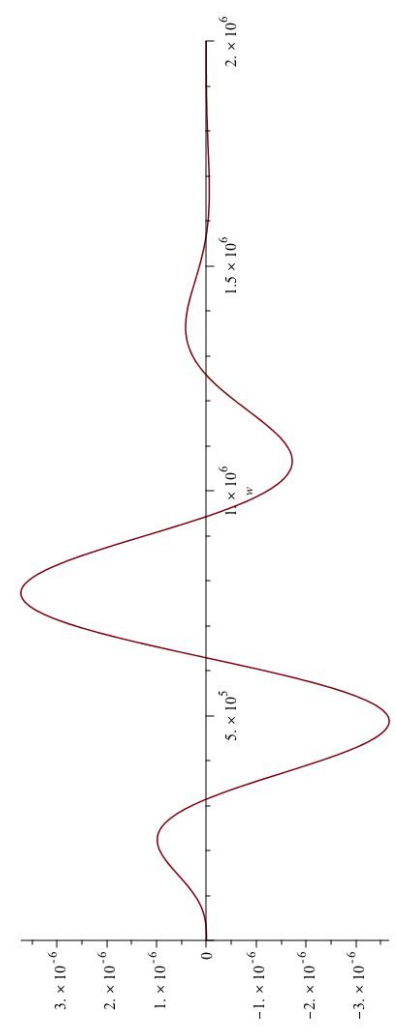

(b) Imag. part of $\mathcal{F}\{g\}(\omega)$

Figure 2: Spectral content of $g(t)$ 


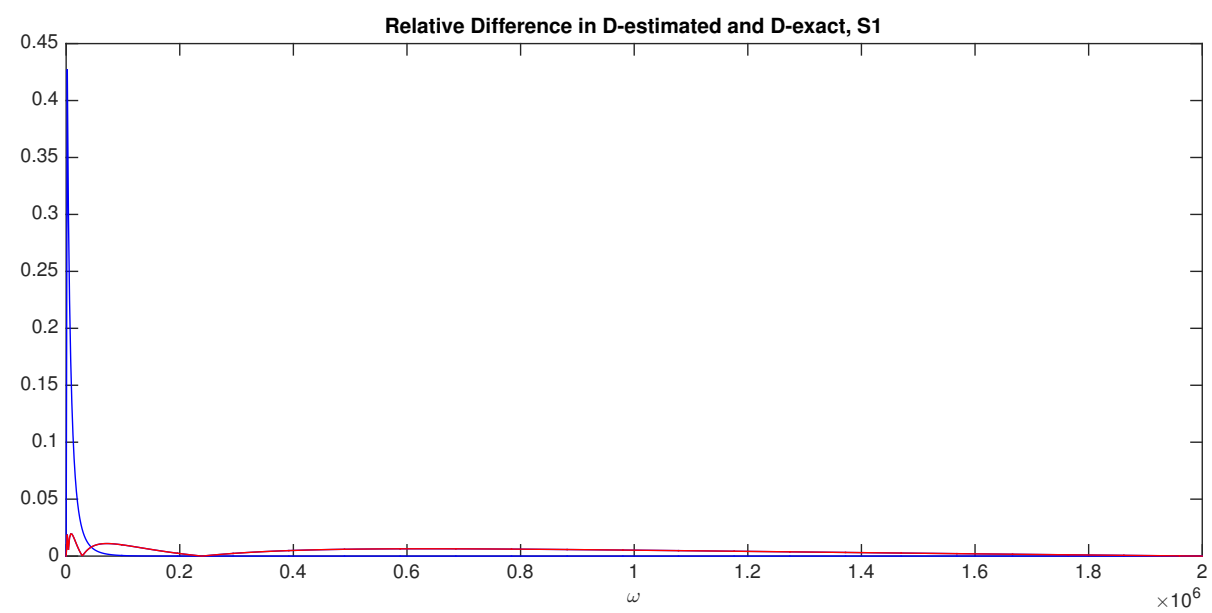

(a) $\mathrm{S} 1$

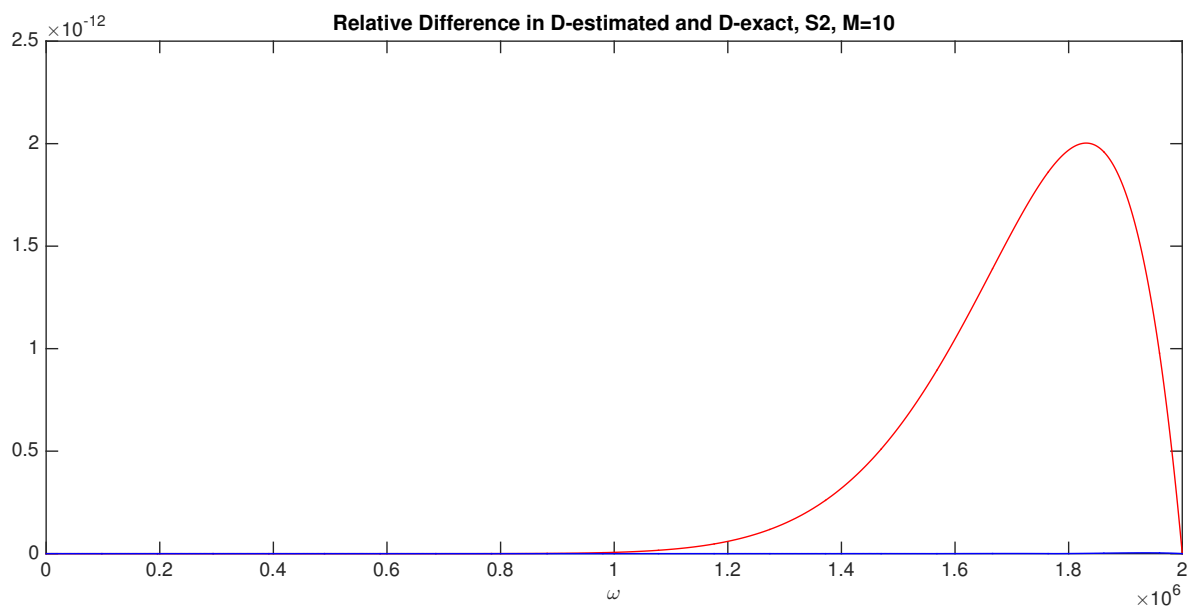

(b) S2

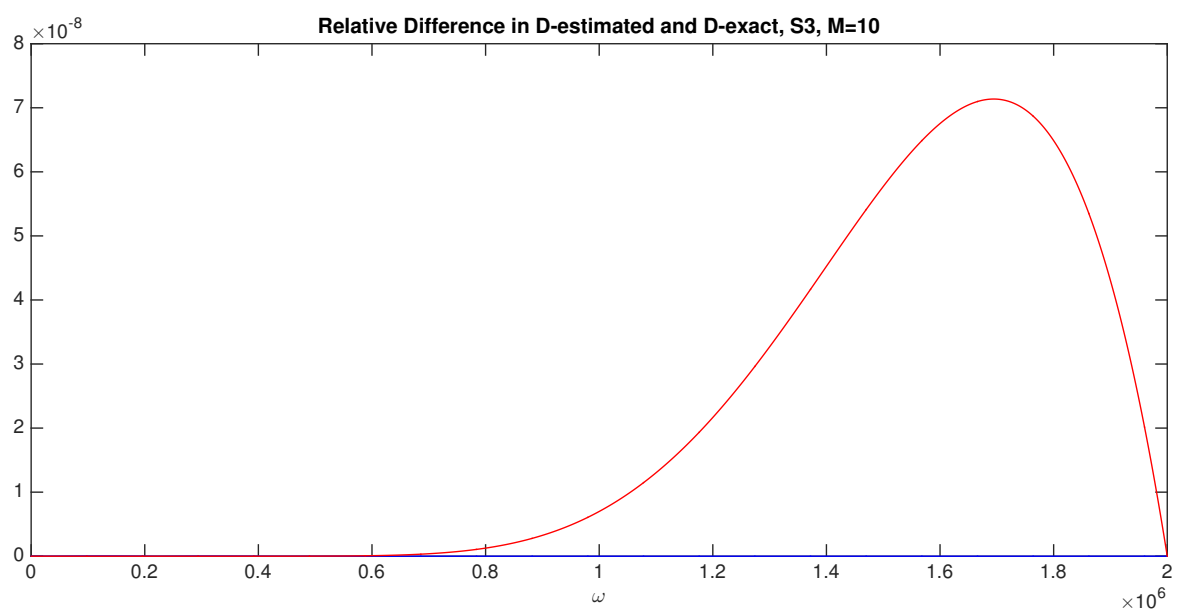

(c) $\mathrm{S} 3$ 


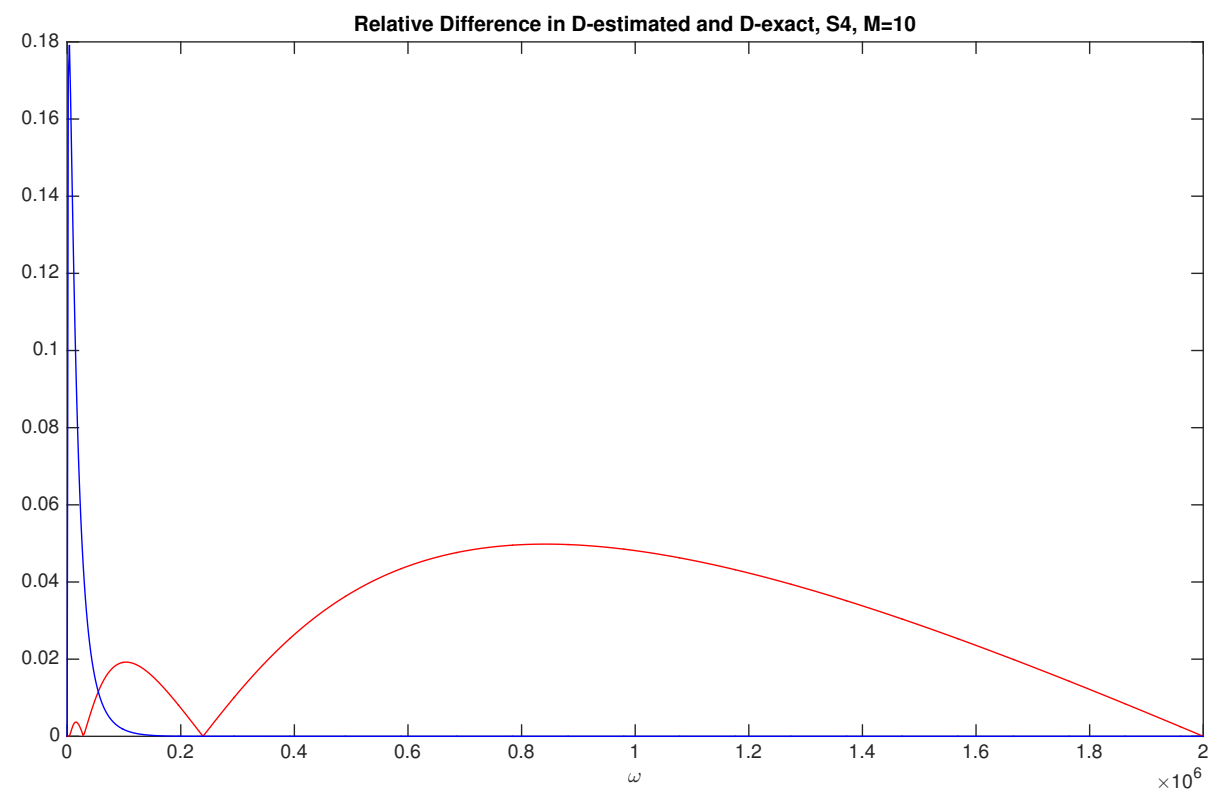

(d) $\mathrm{S} 4$

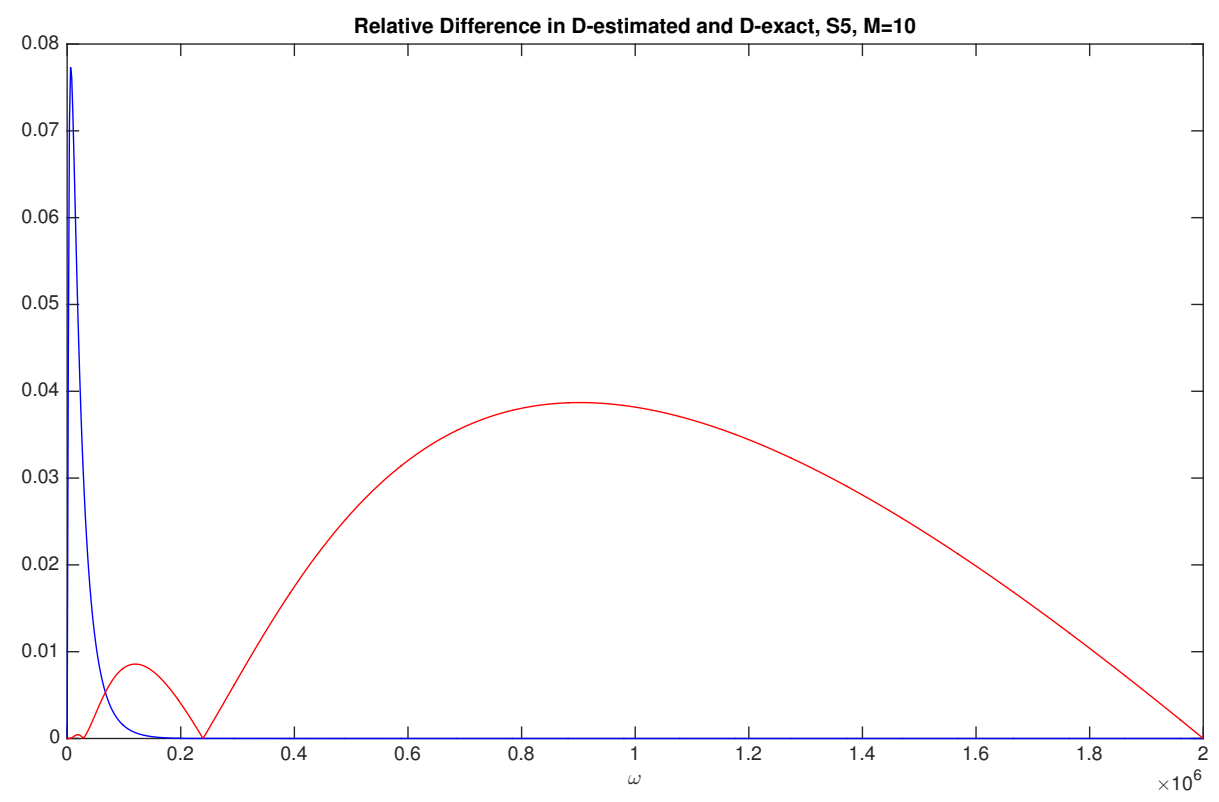

(e) S5

Figure 3: Comparison of relative errors with $M=10$ for S1 to S5. Blue: Equally spaced grids, Red: logdistributed grids 


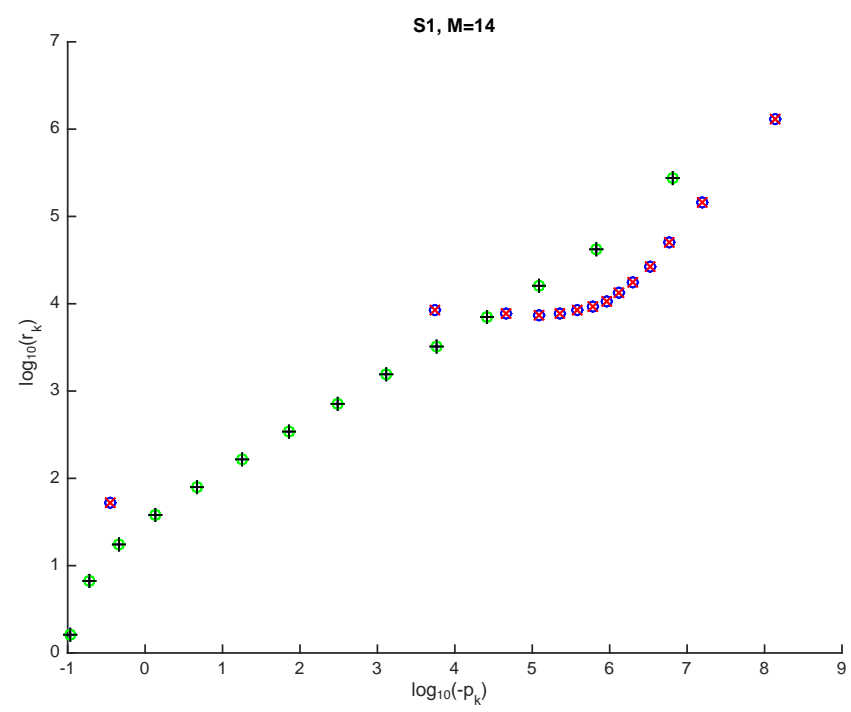

Figure 4: $\left(\log _{10}\left(-p_{k}\right), \log _{10}\left(r_{k}\right)\right), k=1, . ., 14$. Blue circle: Approach 1 with Equally-spaced grids, Red $\mathrm{x}$ : Approach 2 with Equally-spaced grid, Green circle: Approach 1 with Log-spaced grids, Black +: Approach 2 with Log-spaced grids

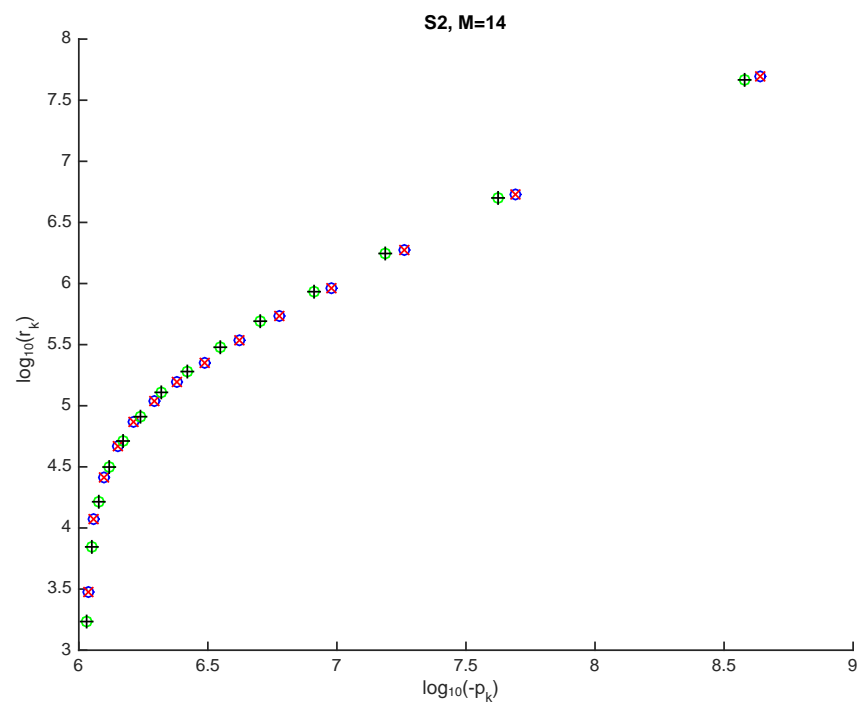

Figure 5: $\left(\log _{10}\left(-p_{k}\right), \log _{10}\left(r_{k}\right)\right), k=1, . ., 14$. Blue circle: Approach 1 with Equally-spaced grids, Red x: Approach 2 with Equally-spaced grid, Green circle: Approach 1 with Log-spaced grids, Black +: Approach 2 with Log-spaced grids 


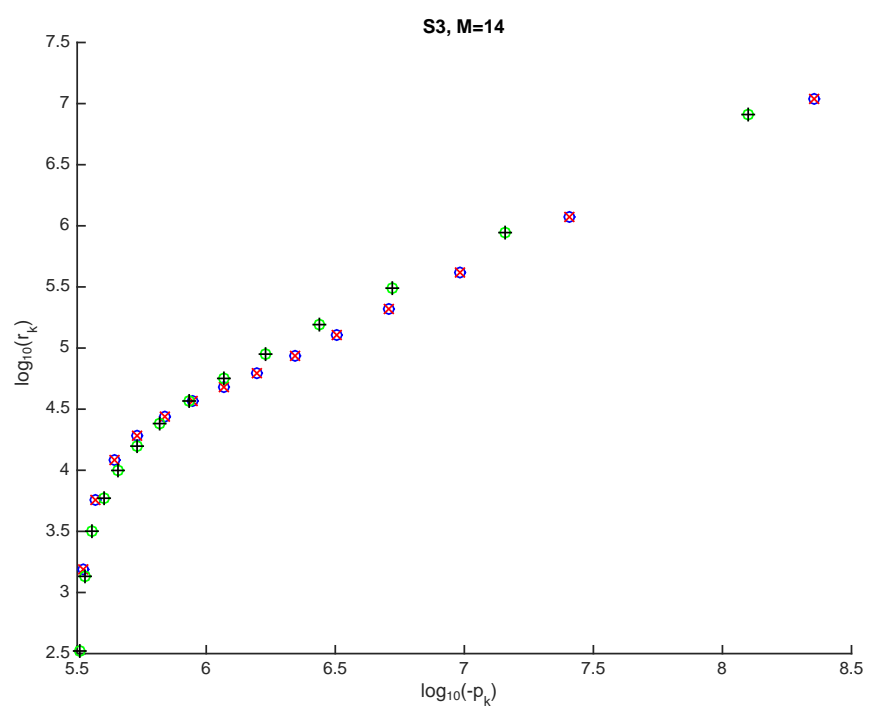

Figure 6: $\left(\log _{10}\left(-p_{k}\right), \log _{10}\left(r_{k}\right)\right), k=1, . ., 14$. Blue circle: Approach 1 with Equally-spaced grids, Red $\mathrm{x}$ : Approach 2 with Equally-spaced grid, Green circle: Approach 1 with Log-spaced grids, Black +: Approach 2 with Log-spaced grids

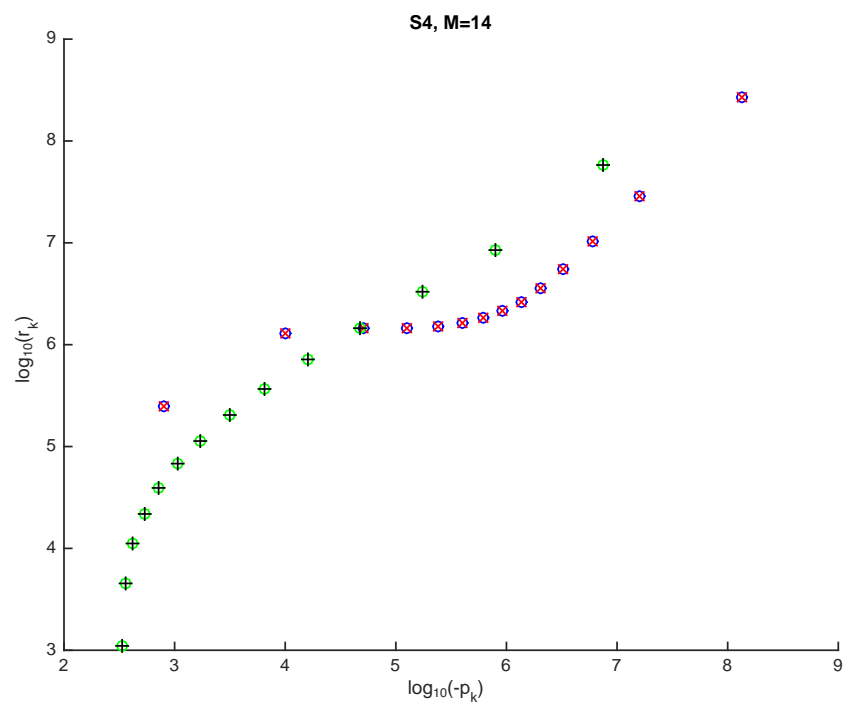

Figure 7: $\left(\log _{10}\left(-p_{k}\right), \log _{10}\left(r_{k}\right)\right), k=1, . ., 14$. Blue circle: Approach 1 with Equally-spaced grids, Red $\mathrm{x}$ : Approach 2 with Equally-spaced grid, Green circle: Approach 1 with Log-spaced grids, Black +: Approach 2 with Log-spaced grids 


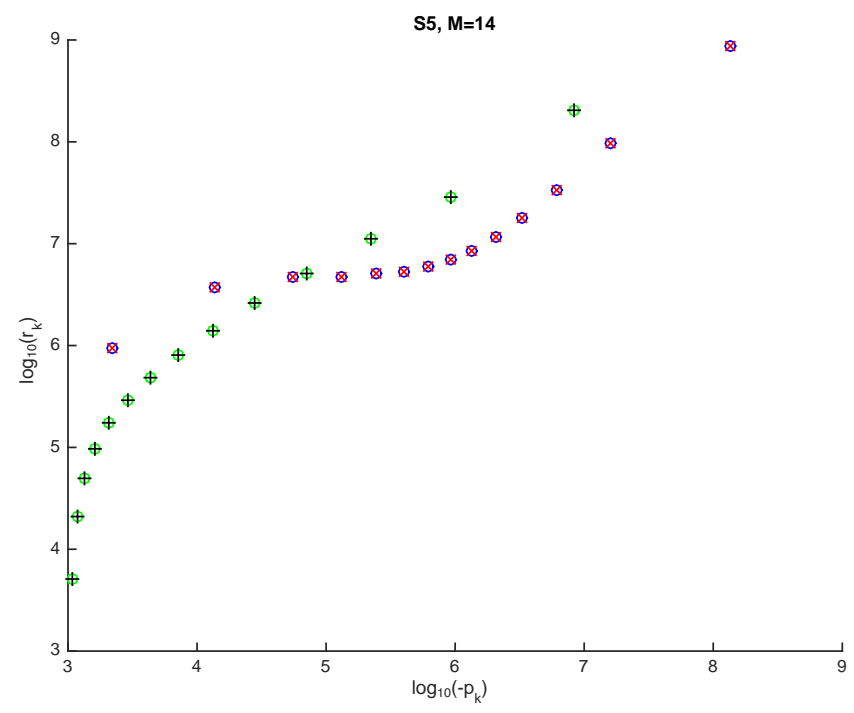

Figure 8: $\left(\log _{10}\left(-p_{k}\right), \log _{10}\left(r_{k}\right)\right), k=1, . ., 14$. Blue circle: Approach 1 with Equally-spaced grids, Red $\mathrm{x}$ : Approach 2 with Equally-spaced grid, Green circle: Approach 1 with Log-spaced grids, Black +: Approach 2 with Log-spaced grids

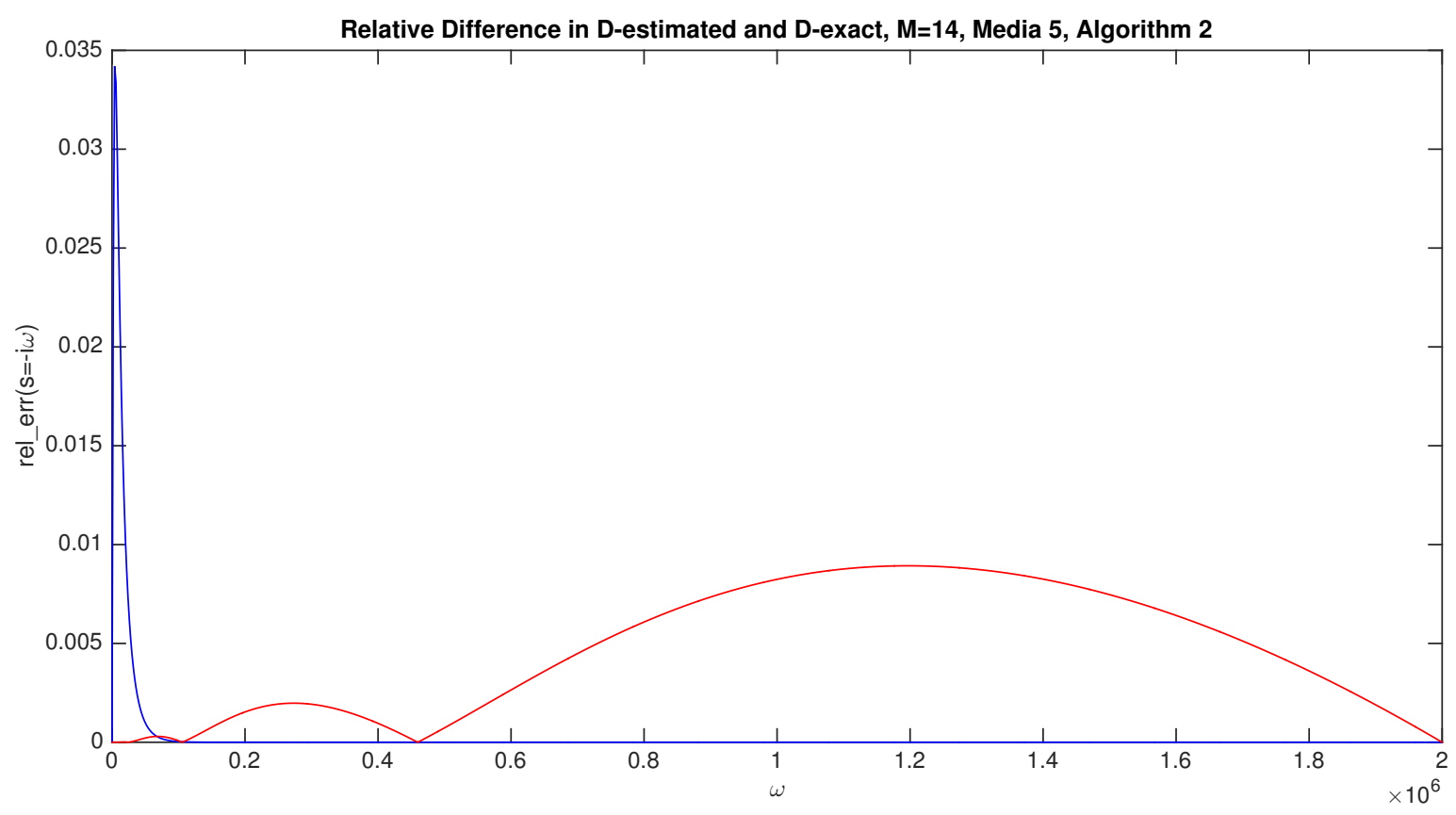

Figure 9: $r e l \_e r r(s=-i \omega)$, Blue: equally spaced grids, Red: log-distributed grids 


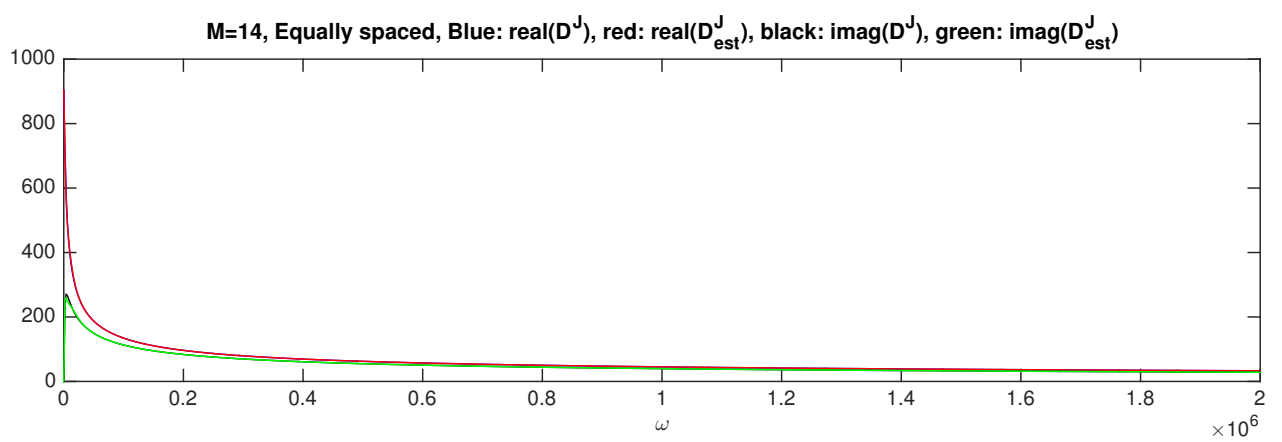

(a) Equally spaced grids

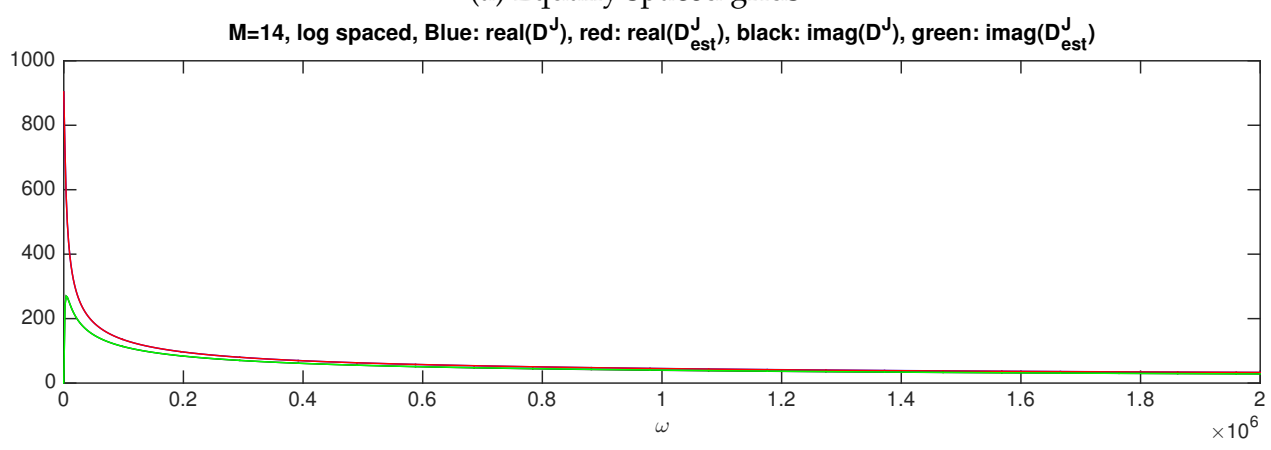

(b) log-distributed grids

Figure 10: Comparison of $D^{J}(s=-i \omega)$ and $D_{\text {est }}^{J}(s=-i \omega)$. Blue: $\operatorname{real}\left(D^{J}\right)$, Red: $\operatorname{real}\left(D_{\text {est }}^{J}\right)$, Black: $\operatorname{imag}\left(D^{J}\right)$, Green: $\operatorname{imag}\left(D_{\text {est }}^{J}\right)$ 


\section{References}

[1] Daniel Alpay, Joseph A Ball, Israel Gohberg, and Leiba Rodman. The two-sided residue interpolation in the Stieltjes class for matrix functions. Linear algebra and its applications, 208:485-521, 1994.

[2] Athanasios C. Antoulas, Sanda Lefteriu, and A. Cosmin Ionita. A tutorial introduction to the Loewner framework for model reduction. In Model reduction and approximation, volume 15 of Comput. Sci. Eng., pages 335-376. SIAM, Philadelphia, PA, 2017.

[3] J-P Berrut and Hans D Mittelmann. Lebesgue constant minimizing linear rational interpolation of continuous functions over the interval. Computers \& Mathematics with Applications, 33(6):77-86, 1997.

[4] M.A. Biot. Theory of propagation of elastic waves in a fluid-saturated porous solid. I. Low-frequency range. The Journal of the Acoustical Society of America, 28:168, 1956.

[5] M.A. Biot. Theory of propagation of elastic waves in a fluid-saturated porous solid. II. Higher frequency range. The Journal of the Acoustical Society of America, 28(2):179-191, 1956.

[6] M.A. Biot. Mechanics of deformation and acoustic propagation in porous media. Journal of applied physics, 33(4):1482-1498, 1962.

[7] Emilie Blanc, Guillaume Chiavassa, and Bruno Lombard. Wave simulation in 2d heterogeneous transversely isotropic porous media with fractional attenuation: A cartesian grid approach. Journal of Computational Physics, 275:118-142, 2014.

[8] G. Blance, G. Chiavassa, B. Lombard, A time-domain numerical modeling of two-dimensional wave propagation in porous media with frequency-dependent dynamic permeability, J. Acoust. Soc. Am. 134(6)(2013) 4610-4623.

[9] J.M. Carcione. Wave Fields in Real Media: Wave Propagation in Anisotropic, Anelastic and Porous Media. Pergamon-Elsevier, Oxford, 2001.

[10] John W. Dettman. Applied Complex Variables. Dover publications, 1965.

[11] Mohamed Fellah, Zine El Abiddine Fellah, Farid G Mitri, Erick Ogam, and C Depollier. Transient ultrasound propagation in porous media using biot theory and fractional calculus: Application to human cancellous bone. The Journal of the Acoustical Society of America, 133(4):1867-1881, 2013.

[12] E. Blance, G. Chiavassa, B. Lombard, Wave simulation in 2D heterogeneous transversely isotropic porous media with fractional attenuation: a Cartesian grid approach. J. Comput. Phys. 275 (2014), 118-142. 76S05 (82B43)

[13] J. F. Lu and A. Hanyga, Wave field simulation for heterogeneous porous media with singular memory drag force, J. Comput. Phys. 208(2)(2005), pp. 651-674.

[14] Y. J. Masson and S. R. Pride, Finite-difference modeling of Biot's poroelastic equations across all frequencies, Geophys., 75(2)(2010), pp. N33-N44

[15] Multiprecision Computing Toolbox for MATLAB 4.4.7.12736. Advanpix LLC., Yokohama, Japan, 2018.

[16] JK Gelfgren. Multipoint padé approximants converging to functions of stieltjes' type. In Padé Approximation and its Applications Amsterdam, pages 197-207. Springer, 1981.

[17] A Hosokawa. Ultrasonic pulse waves in cancellous bone analyzed by finite-difference time-domain methods. Ultrasonics, 44:e227-e231, 2006.

[18] D.L. Johnson, J. Koplik, and R. Dashen. Theory of dynamic permeability and tortuosity in fluidsaturated porous media. Journal of fluid mechanics, 176(1):379-402, 1987.

[19] Yuji Nakatsukasa, Olivier Sète, and Lloyd N Trefethen. The AAA algorithm for rational approximation. arXiv preprint arXiv:1612.00337, 2016.

[20] Miao-Jung Yvonne Ou. On reconstruction of dynamic permeability and tortuosity from data at distinct frequencies. Inverse Problems, 30(9):095002, 2014. 